\title{
Itinerant routing in elastic optical networks
}

\author{
Ireneusz Szcześniak, Artur Gola, Andrzej Jajszczyk, Andrzej R. Pach, and Bożena Woźna-Szcześniak
}

\begin{abstract}
We state a new problem of itinerant routing in elastic optical networks, which we define as the establishment and reconfiguration of an itinerant connection. In an itinerant connection, one of the connection end nodes is allowed to change. Itinerant routing could also be considered a form of connection restoration, where a connection has to be restored to a different end node. With the introduction of new mobile-network architectures, the progressing integration of wireless and optical networks, the continuing growth of wireless high-bitrate services, and the eventual deployment of elastic optical networks, there is a need to support this itinerant routing in the elastic optical networks. We present and discuss two major use cases of the itinerant routing, and propose a novel reconfiguration algorithm. Our algorithm does not deteriorate the network performance, and requires half as many new links to configure as the shortestpath reconfiguration algorithm. The performance evaluation was carried out with $\mathbf{4 6 8 0 0}$ simulation runs using randomly-generated realistic transport networks.
\end{abstract}

Index Terms-elastic optical networks, optical transport, 5G, wireless access networks, itinerant routing, spectrum fragmentation, mobile backhaul

\section{INTRODUCTION}

The wireless access network traffic has increased manyfold in recent years along with the number of users, and network operators are expecting its further dynamic increase which has to be serviced by the optical transport network [2]. Since this trend is expected to continue, the network densification across all network layers is regarded as a key and inevitable enabler of the fifth generation mobile $(5 \mathrm{G})$ networks [3], putting higher requirements on the optical transport network.

Network densification is a major reason why optical transport networks should continue to grow in the metropolitan area networks (MANs). Currently the Optical Transport Network (OTN) is deployed in MANs over wavelength-division multiplexing (WDM) networks [4]. Even though OTN was designed for long-haul communication, it is also deployed in MANs because it can economically switch a large number of subwavelength short-distance circuits.

The elastic optical networks (EONs) are very likely to be deployed because they utilize the limited erbium window more efficiently than the incumbent WDM networks [5]. The flex-grid has now been standardized with $6.25 \mathrm{GHz}$ channel spacing [6], but as the technology is deployed and further improved, we can expect smaller channel spacing in the future,

This work was supported by the postdoctoral fellowship number DEC2013/08/S/ST7/00576 from the Polish National Science Centre. The simulation results were obtained using PL-Grid, the Polish supercomputing infrastructure. This is the extended version of a conference paper [1].

I. Szcześniak is with the Institute of Computer and Information Sciences of the Częstochowa University of Technology, Częstochowa, Poland.

A. Gola and B. Woźna-Szcześniak are with the Institute of Mathematics and Computer Science of the Jan Długosz University, Czestochowa, Poland.

A. Jajszczyk and A. R. Pach are with the Department of Telecommunications of the AGH University of Science and Technology, Krakow, Poland. supporting lower client bitrates, which would increase network utilization, lower blocking probability and lower spectrum fragmentation. For even lower bitrates, OTN over the EONs is an option [7].

The centralized radio access network (C-RAN, also called the cloud RAN) is a major candidate for a $5 \mathrm{G}$ network architecture [8]. In the C-RAN, the base station (BS) is kept simple to allow for a large-scale deployment of inexpensive small cells (picocells or femtocells), while the data processing is relegated to various data centers (DCs), which allows for the load balancing of radio signal processing, the network function virtualization (NFV), and easier hardware upgrades. However, the C-RAN requires agile high-bitrate connections between BSs and DCs for transporting digitized radio signals according to bandwidth-demanding standards like, most notably, the Common Public Radio Interface (CPRI). The ambitious operation of the C-RAN, and quite likely of other $5 \mathrm{G}$ architectures, is termed the wireless-optical network orchestration, and is being enabled by the first commercial silicon photonic integrated circuits [2]. The wireless-optical network orchestration requires the support of itinerant routing in the EONs.

There are a number of novel $5 \mathrm{G}$ use cases, where the capacity provided by the transport network should migrate between BSs to efficiently utilize the transport capacity. For example, to provide service in buses and trains, the moving femtocell (MFC) [9] migrates between BSs, and along with it the capacity should migrate too. Likewise, moving crowds and the emergency vehicles also require the capacity to migrate between BSs. These mobile high-bitrate wireless services require the support of itinerant routing in the EONs.

Our contribution is the statement of the new research problem of itinerant routing in the EONs, and its solution: a novel reconfiguration algorithm. We study the algorithm performance in comparison with the shortest-path reconfiguration. This paper is an extension of a conference paper [1], where we introduced the itinerant routing, though we initially called it the mobile routing. However, we renamed it to itinerant routing, not to confuse it with routing in mobile networks.

We carry out the simulative performance evaluation of the reconfiguration algorithms with three algorithms for finding candidate paths, and three policies of allocating spectrum. The two metrics of chief interest compared are the number of new links to configure, and the probability of reconfiguring a connection, along with a number of other metrics which augment the comparison.

The paper is organized as follows. In the following section we review key related works. In Section [II we state the research problem, in Section 1 we describe the proposed algorithm, and in Section $\mathrm{V}-\mathrm{B}$ we report the obtained performance evaluation results. Section $\mathrm{VI}$ concludes the paper. 


\section{RELATED WORKS}

This paper differs from [1] in a number of ways. First, we extend the study of itinerant routing, so that candidate paths are computed not only with the adapted and constrained Dijkstra algorithm for the EONs [10], but also with two other algorithms frequently used: the Yen K-shortest paths (Yen-KSP) algorithm, and the link-disjoint all shortest paths (LD-ASP) algorithm. Second, we replace a complicated traffic model with a simple and tractable one. Next, we extended the study to include the random spectrum allocation policy. Finally, we remove the incremental reconfiguration algorithm from the study, since we have already shown it is markedly inferior, and since its performance results would only obfuscate the study.

Reconfiguration in itinerant routing is a form of routing and spectrum assignment (RSA) in EONs. Several variations of the RSA problem in EONs have been well studied, both for many demands at once and for a single demand using the integer linear programming and heuristic algorithms [11], [12]. The RSA problem has been tackled in various contexts, e.g., grooming or virtual network embedding, and to the best of our knowledge, the end nodes of a demand in these problems do not change, while in our case they do.

In [13] the authors argue that the quality of service (QoS) of optical connections does not take into account the parameters of the connection setup, rerouting or tear down, such as the preemption probability, and therefore introduce the grade of service (GoS) for optical connections to address these parameters. Our work contributes new results on the GoS of itinerant routing in elastic optical networks.

The research on mobile backhaul usually concentrates on PONs which integrate the wireless access network with the optical transport network [14]. We, however, concentrate on the support of itinerant routing in the EONs required by the mobile backhaul, which will further gain in importance if the mobile backhaul becomes elastic.

In [15] the authors propose the concept of the moving extended cell (MEC), which guarantees the high-bitrate connectivity for a highly mobile client by transceiving wireless signal for this client also in the picocells neighboring the picocell in which the client currently resides. Our algorithm can be used to reconfigure quickly a high-bitrate connection when the client roams between picocells serviced by different hotels.

Our research results are well suited for radio-over-fiber (RoF) mobile backhaul which amalgamates the optical and wireless networks by transceiving subcarriers [16]. In [17] the authors propose a cost-effective RoF architecture which converts the $60 \mathrm{GHz}$ radio subcarriers directly into the subcarriers of the optical erbium window, thus enabling the elastic optical networks to make inroads into mobile backhaul.

We note that itinerant routing is not the anycast routing, though it bears some resemblance. In the anycast routing, commonly used in the Internet, the destination node of a connection can be any from a given set, but once the connection is established, the destination node does not change. The hallmark of the itinerant routing is that the end nodes can change during the duration of the connection.

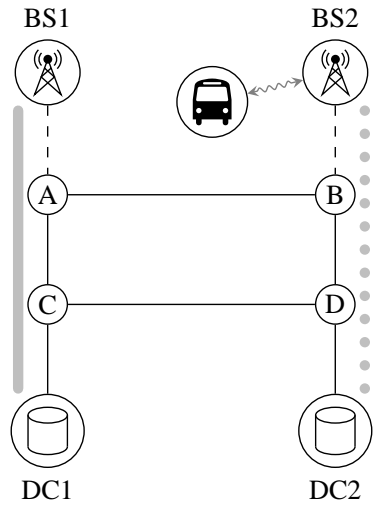

(a) before reconfiguration

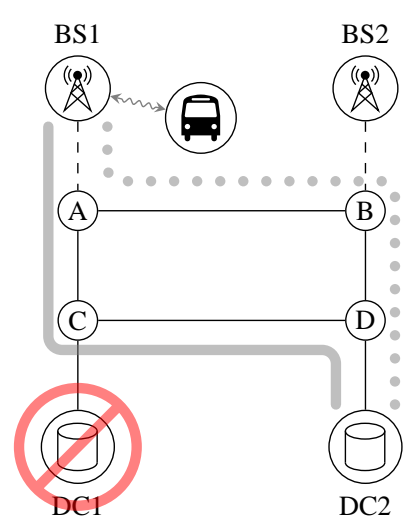

(b) after reconfiguration
Fig. 1: Two use cases of itinerant routing, where is a base station, $\Theta$ is a data center, $\theta$ is a means of public transport, and $\mathrm{A}, \mathrm{B}, \mathrm{C}$, and $\mathrm{D}$ are the EON nodes.

\section{Problem Statement}

We model the EONs by an undirected graph $G(V, E)$, where $V$ is the set of nodes, and $E$ is the set of links. The links have the attributes of length and available slices. Optical spectrum is divided into slices of equal bandwidth, with $\Omega$ being the set of all slices. A given number of contiguous slices create a slot, which can be used to transport an optical signal. A bidirectional unicast symmetric connection is established by allocating the same slices on undirected links.

We introduce a new concept of the itinerant connection, where one of the connection end nodes is allowed to change during the duration of the connection. A connection is established between the source node $s$ and the destination node $t$ with the set of contiguous slices $S$ along the path $P$. The problem is to find a reconfiguration path $P^{\prime}$, and a set of reconfiguration slices $S^{\prime}$, when the connection has to be reconfigured for the new destination node $t^{\prime}$. The problem of reconfiguring the connection for a new source node $s^{\prime}$ is the same.

There are two major use cases of an itinerant connection in 5G networks: 1) a connection is switched to a different DC, and 2) a connection is switched to a different BS. These use cases are illustrated in Fig. 1, where the connections before the reconfiguration are shown in Fig. 1a, and after in Fig. 1b

In the first use case, a connection from a BS is switched to a new DC, because the current DC is overloaded or went down, or the new DC offers a better deal. As shown in Fig. 1 the connection from BS1 is switched from DC1 to DC2.

In the second use case, a connection from a DC is switched to a new BS, because an MFC or a crowd roams to this BS. As shown in Fig. 1, the MFC of a bus makes a transition from $\mathrm{BS} 2$ to $\mathrm{BS} 1$, and the transport capacity migrates along, i.e., the connection from DC2 is switched from BS2 to BS1.

In general, the problem is how to reconfigure the connection fast and frugally. We want to minimize both the reconfiguration time and the resources used, but there is a trade-off between them in that the faster we want to reconfigure the connection, the more resources we might need to spend. 


\section{PROPOSED ALGORITHM}

Specifically, we concentrate on the number of link configurations, since they require substantial time, and can cause service disruptions. Limiting the number of the required link configurations would also limit the control traffic in the network, and limit the network destabilization.

A natural solution to the problem is to tear down the established connection, and search for a new path from the source node to the new destination node $t^{\prime}$ with any available slices capable of supporting a demand. We call it the complete reconfiguration.

However, we propose a reconfiguration algorithm, which minimizes the number of the required new links by reusing the links of the already established connection. The algorithm attempts to optimally bridge one of the nodes of the already established connection to the new destination node. The algorithm could be implemented in the path computation element (PCE) of the Generalized Multiprotocol Label Switching (GMPLS) control plane.

For every node $n$ of the path $P$ of the established connection, the algorithm searches for a shortest bridging path $B_{n}$ from a node $n$ to the new destination node $t^{\prime}$, provided the bridging path meets the spectrum contiguity and continuity constraints, i.e., it has to use on all its links the same set of contiguous slices $S$ already used by the established connection. Having a bridging path found, a candidate reconfiguration path $P_{n}$ is produced by concatenating $C_{n}$ and $B_{n}$, where $C_{n}=\operatorname{CUT}(P, n)$ is the front of the path $P$ cut at a node $n$, as returned by the function $\operatorname{CUT}(P, n)$.

Candidate reconfiguration paths are pushed into the priority queue $Q$, where they are sorted in ascending order first by the number of the new links they require (i.e., by the number of links in $B_{n}$ ), and next by the number of all their links, new or reused (i.e., by the number of links in $P_{n}$ ). The number of candidate reconfiguration paths pushed into $Q$ equals to at most the number of nodes in the path $P$. Once all the candidate paths have been pushed into $Q$, the solution sought is at the front of the queue. If no candidate reconfiguration path is found with bridging, the algorithm resorts to the complete reconfiguration.

The shortest bridging paths belong to the reverse shortestpath tree (SPT) rooted at the new destination node $t^{\prime}$. The paths in a reverse SPT end at the root, and lead from other nodes, as opposed to an SPT whose paths start at the root, and lead to other nodes. We get a reverse SPT by reversing an SPT obtained for $G^{R}$, the reverse graph of $G$. For an undirected graph $G$, an SPT and its reverse SPT are the same, but we keep the distinction anyway to make the algorithm applicable to directed graphs too.

We grow an SPT using our shortest-path algorithm for the EONs [10]: an adapted and constrained Dijkstra, which takes into account the spectrum continuity and contiguity constraints. The algorithm finds all available slices in the SPT. The objective of the algorithm is to find a shortest path to a given node (or a given set of nodes), and to this end the algorithm builds an SPT. To grow a minimal SPT, the algorithm stops when all the nodes of the established connection are reached.
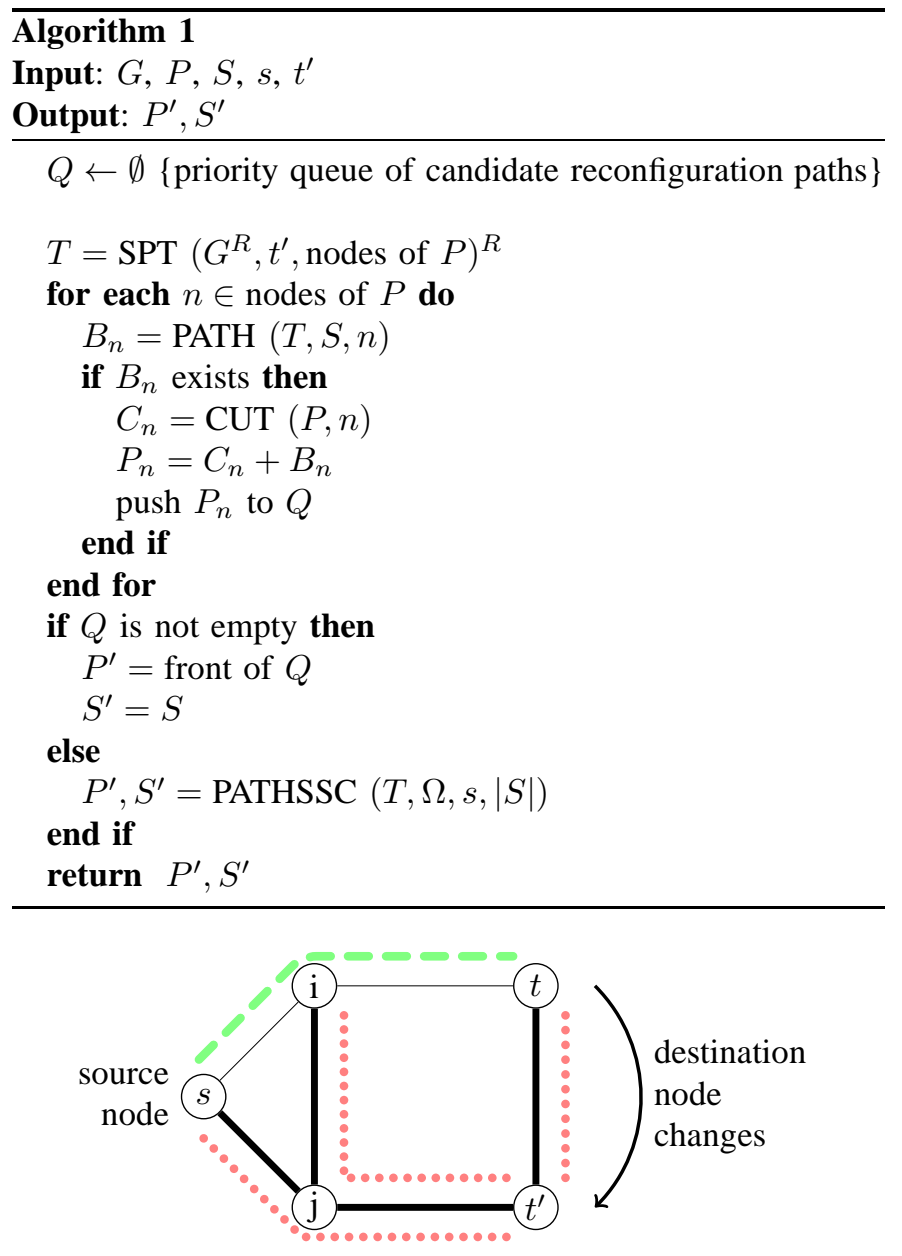

Fig. 2: Reconfiguration example, where - is the established connection, - are the links of the shortest-path tree, and $\ldots . .$. are the candidate bridging paths rooted in $t^{\prime}$.

Algorithm 1 displays the proposed reconfiguration algorithm. The algorithm takes as input the graph $G$, the path $P$, the set of slices $S$, the source node $s$, and the new destination node $t^{\prime}$, and produces the reconfiguration path $P^{\prime}$ and the reconfiguration set of slices $S^{\prime}$. If no solution can be found, $P^{\prime}$ and $S^{\prime}$ are returned null.

The algorithm produces the bridging paths $B_{n}$ from the reverse SPT $T$, obtained by reversing the SPT returned by the SPT function. The function PATH $(T, S, n)$ returns a path from a node $n$ in the reverse SPT $T$, provided the path can support exactly the set of slices $S$. The function PATHSSC $(T, s,|S|)$ returns a pair of a path from the node $s$ in the reverse SPT $T$, and a set of $|S|$ contiguous slices, where $|S|$ is the cardinality of $S$. The returned set of contiguous slices is selected from the set of slices available along that path according to an employed spectrum selection policy.

Figure 2 shows a reconfiguration example. A connection $(-\infty)$ is established from $s$ to $t$, and the destination node changes to $t^{\prime}$. A reverse SPT is built (-), and candidate bridging paths $(\cdots \cdots)$ are produced.

As reported in [10], the optimal shortest-path search can take even a second, which is too long for a network to react in real time. Therefore, we propose that the reconfiguration paths 
are calculated in advance for the foreseeable reconfigurations.

\section{Simulative STUdy}

We study with simulations the performance of the proposed algorithm in comparison with the complete reconfiguration. We are mainly interested in the number of new links required during the connection reconfiguration, but we also mind other performance metrics, to ensure the proposed algorithm does not impede the network performance in general. The performance of the two reconfiguration algorithms is evaluated for various loads, and compared for three routing algorithms, and three spectrum allocation policies. We do not use a single network topology, like NSFNet, but instead a hundred of randomly-generated realistic network topologies.

A routing algorithm is responsible for finding a path for a given demand. In our simulations, a routing algorithm is used in three cases: 1) to establish a connection, 2) to find a path for the complete reconfiguration, and 3 ) to find bridging paths.

The three routing algorithms compared are as follows: optimal, Yen-KSP, and LD-ASP. The optimal routing algorithm finds a shortest-path (using the algorithm in [10]), while the Yen-KSP and LD-ASP algorithms do not guarantee that the path they find is the shortest, since they are heuristic algorithms. These routing algorithms search for a path with the largest set of slices, capable of supporting at least a given demand. From that largest set of slices, we select the contiguous slices according to the spectrum allocation policy. For a given generated graph, in the routing algorithms we do not consider paths which are longer than twice the length of the longest shortest-path in the graph.

The Yen-KSP routing algorithm finds at most K-shortest paths with the Yen algorithm, and then selects the shortest one which can support a given demand. We search for $K=10$ paths. The LD-ASP routing algorithm finds all link-disjoint paths by repeatedly searching for a shortest path with the links used by previous shortest paths disabled until no path can be found. From among the paths found, the shortest one capable of supporting a demand is selected.

In the simulations we also evaluate the performance of the reconfiguration algorithms when the Yen-KSP and LDASP algorithms are harnessed. The proposed reconfiguration algorithm efficiently gets all the bridging paths from a reverse SPT obtained once with the optimal routing algorithm, but it can also get the bridging paths separately with the Yen-KSP and LD-ASP algorithms.

From the set of slots found, we choose exactly the number of requested contiguous slices according to one of three policies: first, fittest, or random. In the first available policy, the slices with the lowest number are chosen. In the fittest available policy, the slot with the lowest number of slices are chosen which still can handle the demand. In the random policy, the slices are chosen at random.

The demand arrival time follows the exponential distribution with the mean of $\lambda$ hours. The end nodes of a demand are different and chosen at random. A connection is reconfigured only once after it is established. The holding time follows the exponential distribution with the mean of $\beta$ hours, after which the connection is torn down. We set the mean holding time $\beta=10$ hours.

Furthermore, the number of slices of a demand is constant throughout the duration of the connection and follows the distribution of (Poisson $(\gamma-1)+1)$ with the mean of $\gamma$, i.e., the Poisson distribution shifted by one to the right, to avoid getting zero for the number of slices. We set $\gamma=10$.

The new destination node $t^{\prime}$ is chosen at random in such a way that the number of hops it is away from the destination node $t$ follows the (Poisson $(0.5)+1)$ distribution. We add one to the Poisson distribution to avoid getting zero, which would result in the destination node $t^{\prime}=t$. This way of choosing $t^{\prime}$ allows us to model the connections being frequently switched to a new BS (which are close to the node $t$ ), and infrequently switched to a new DC (which are farther from the node $t$ ).

We define the offered load $\mu$ as the ratio of the number of slices demanded to the number of slices in the network. We vary the offered load $\mu$ with 26 values of $0.1,0.15, \ldots, 0.7,0.8, \ldots, 2.0$. We express the mean demand arrival time $\lambda$ as a function of the offered load $\mu$ as given by (1), where $\alpha$ is the mean number of links of all shortest-paths in a network begin simulated. We keep the mean connection holding time $\beta$ constant. The average number of demanded slices is $\alpha \beta \gamma / \lambda$, since the intensity of a demand arrival is $1 / \lambda$, and since in an unloaded network a connection takes on average $\alpha \gamma$ slices over the mean holding time of $\beta$. The number of slices in the network is $|E||\Omega|$, and so the offered load $\mu=\alpha \beta \gamma /(\lambda|E||\Omega|)$, from which (1) follows.

$$
\lambda(\mu)=\frac{\alpha \beta \gamma}{\mu|E||\Omega|}
$$

We define network utilization as the ratio of the number of the slices in use to the total number of slices on all edges. We cannot directly control the network utilization, but measure it in response to the offered load $\mu$.

In the simulations we generated random Gabriel graphs, which can be used to model mesh transport network topologies [18]. Gabriel graphs can capture the properties of mesh transport network topologies in that they are planar, model well the length of fiber cables, the node degrees, and the biconnected property. We efficiently generate Gabriel graphs by first using the Delaunay triangulation, and then removing those links which violate the Gabriel graph properties.

The complete reconfiguration gives a chance to defragment the optical spectrum, as the connection is completely torn down and established anew with potentially different slices. If the new connection uses different slices than the previously established connection, all the links of the new path have to be configured. However, if the new connection uses the same slices, then only the links not present in the already established connection have to be configured, thus reducing the number of the required link configurations.

We developed and ran the software simulator under Linux within PL-Grid, the Polish supercomputing infrastructure. We wrote the simulator in $\mathrm{C}++$ with the Boost libraries, but without a simulation framework, like OMNet++, as most of the functionality needed is already provided by Boost, such as the graph manipulation with the Boost Graph Library, or the 
TABLE I: Statistics of the generated Gabriel networks.

\begin{tabular}{|l|r|r|r|r|}
\hline value & min & average & max & variance \\
\hline Number of links & 160 & 179.13 & 194 & 48.47 \\
Link length & 1 & 97.91 & 347 & 2695.48 \\
Node degree & 1 & 3.583 & 8 & 1.221 \\
SP length & 1 & 589.45 & 1582 & 78103.1 \\
SP hops min & 1 & 6.7667 & 22 & 10.8898 \\
\hline
\end{tabular}

measurement accumulation with the Boost Accumulator. The software is available at [19].

\section{A. Runs and populations}

The objective is to produce credible simulation results, which allow us to compare the performance of the proposed reconfiguration algorithm and the complete reconfiguration algorithm. We carry out simulation runs and produce credible means for statistical populations.

A simulation run simulates 100 hours of a network in operation. There are two groups of measurements taken. In the first group, during every simulated hour, the measurements related to connections are taken when the network tries to establish or reconfigure a connection, then the measurements are averaged and reported for that simulated hour. In this group, the following are reported: (a) the probability of establishing a connection, (b) the length of an established connection, (c) the number of slices of an established connection, (d) the probability of reconfiguring a connection, (e) the number of new links of a reconfigured connection, (f) the number of reused links of a reconfigured connection, $(\mathrm{g})$ the length of a reconfigured connection, and $(h)$ the number of slices of a reconfigured connection. In the second group, every simulated hour, the following measurements related to the network are taken: (a) the instantaneous network utilization, (b) the instantaneous number of active connections, and (c) the instantaneous amount of capacity served. The measurements for every hour are averaged and reported as the results of the simulation run.

A statistical population is described by four parameters: the reconfiguration algorithm, the routing algorithm, the spectrum allocation policy, and the offered load $\mu$. We have 468 populations: two reconfiguration algorithms $\times$ three routing algorithms $\times$ three spectrum allocation policies $\times 26$ values of the offered load $\mu$. In a given population, all simulation runs have the same parameters, except the seed of a random number generator in order to generate different Gabriel graphs and different traffic. To get credible results for a population, we carry out 100 simulation runs which are the population samples, and calculate the sample means of the results reported by a simulation run. In total there are 46800 simulation runs (468 populations $\times 100$ samples). We reckon the sample means credibly estimate the means of their populations, since their relative standard error is below $1 \%$.

For the simulation runs of every population, we use the same sample of a hundred different randomly-generated Gabriel graphs. Each graph in the sample has 100 nodes uniformly distributed over an area $1000 \mathrm{~km}$ long and $1000 \mathrm{~km}$ wide, with undirected edges having 400 slices. In generating Gabriel graphs, the number of edges and their length cannot be directly controlled, as it depends on the random location of nodes, and on the candidate edges meeting the conditions of the Gabriel graph. Table \reports the statistics of the generated graphs.

\section{B. Simulation results}

Figures 3 and 4 show the sample means of the measured values as lines, where each line has 26 data points. The figures do not report the standard errors as error bars, because they were too small to be drawn as the aforementioned relative standard errors were below $1 \%$. Most of the sample means are reported as a function of network utilization, and not of the offered load, because we are interested in how the reconfiguration algorithms perform under a given network state expressed by the network utilization.

We do not plot the obtained simulation results for the ED-ASP routing algorithm, because they were worse by a few percent than the results for the Yen-KSP algorithm, and because they would obfuscate the plots. Our general finding is that Yen-KSP outperforms ED-ASP in establishing and reconfiguring a connection, and so we compare the results of the optimal and Yen-KSP routing algorithms only. The optimal routing algorithm performs the best.

Of the central interest is the number of new links required for a reconfigured connection, shown in Fig. 3a. The proposed algorithm requires about half as many new links as the complete reconfiguration, because it reuses more links of the established connection than the complete reconfiguration, as shown in Fig. $3 \mathrm{~b}$ The low number of new reconfiguration links is the reason, we argue, for using the proposed reconfiguration algorithm over the complete reconfiguration algorithm.

The number of all links of a reconfigured connection is a few percent higher for the proposed configuration than for the complete reconfiguration as shown in Fig. 3c, which we show has negligible detrimental effect on the network. The number of links of a reconfigured connection increases up to the network utilization of 0.35 , because the network is able to support circuitous reconfiguration paths, while for the higher network utilization, the longer connections are less likely to be reconfigured, and so the number of all links gets lower.

In Fig. 3. for the proposed reconfiguration algorithm, we show the results for the fittest policy only, because for the first and random policies the algorithm performed comparably, and plotting these results would obfuscate the figures. For the complete reconfiguration algorithm, we also show the results for the random policy, since they are the worst: the algorithm does not try to meet the spectrum continuity constraint, and with the random policy the algorithm is unlikely to reuse the links of the already established connection, especially for the lightly utilized network.

Fig. 4 shows the results for the fittest policy only, because for the first and random policies, the results are worse by a few percent, and reporting them would obfuscate the plots.

Figures $4 \mathrm{a}$, 4b, and $4 \mathrm{c}$ show the results for establishing a connection in gray, and for reconfiguring a connection in black. We are mainly interested in the reconfiguration results, and show the results of establishing connections only to argue that the proposed reconfiguration does not impede the network performance. 


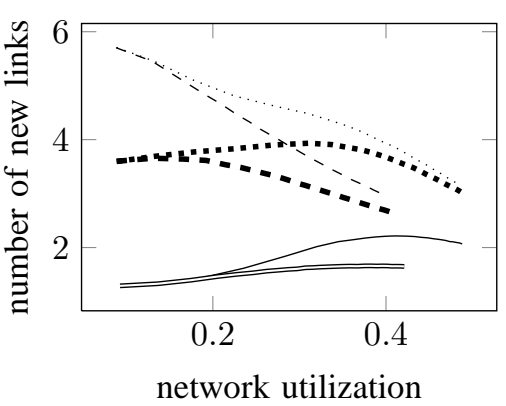

(a) Number of new links.

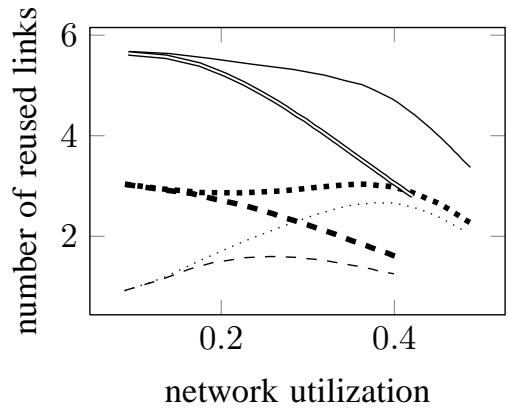

(b) Number of reused links.

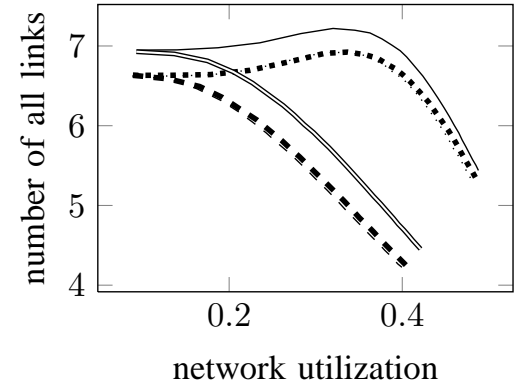

(c) Number of all links.

\begin{tabular}{|c|c|c|}
\hline $\begin{array}{l}\text { proposed, optimal, fittest } \\
\text { proposed, Yen-KSP, fittest }\end{array}$ & $\begin{array}{l}\cdots . \text { complete, optimal, fittest } \\
\text { - - complete, Yen-KSP, fittest }\end{array}$ & $\begin{array}{l}\text { ….... complete, optimal, random } \\
\text { - - complete, Yen-KSP, random }\end{array}$ \\
\hline
\end{tabular}

Fig. 3: The number of new, reused, and all links of a reconfigured connection.

The probabilities of establishing and reconfiguring a connection are shown in Fig. 4a With the optimal routing algorithm employed, the proposed reconfiguration algorithm performs equally well as the complete reconfiguration.

The lengths of established and reconfigured connections are shown in Fig. 4b The length of a reconfigured connection is by a few percent larger for the proposed reconfiguration than for the complete reconfiguration, analogously to the number of all links of a reconfigured connection.

The number of slices of a reconfigured connection is shown in Fig. 4c. As expected, the number of slices gets lower, since the more slices a connection has, the more difficult it is to find a candidate reconfiguration path in an increasingly utilized network. The number of slices of a reconfigured connection in comparison with the established connection is lower because of the reconfiguration case, which requires no slices: the source node is the new destination node.

Fig. 4d shows the network utilization as a function of the offered load $\mu$. The network responds differently to the offered load depending on the way a connection is established, the way a connection is reconfigured, and a number of parameters, e.g., $\beta$. For the optimal routing algorithm, the proposed reconfiguration algorithm utilizes the network comparably with the complete reconfiguration. In comparison with the optimal routing, the Yen-KSP utilizes the network less, as it is less likely to establish and reconfigure the connection, and favors short connections with a small number of slices.

Fig. 4e shows the number of established and reconfigured connections in the network. The proposed reconfiguration algorithm perform slightly worse in comparison with the complete reconfiguration, which is the only detrimental effect of the proposed algorithm we found. Even the Yen-KSP routing algorithm performs well in terms of the number of connections, but its connections are increasingly shorter as the load increases, as shown in Fig. $4 \mathrm{~b}$

The capacity served better captures the network performance, as it takes into account the length of the connections. The capacity served is the sum of the capacities served by every currently established and reconfigured connection. The capacity served by a connection is the product of the number of slices and the path length. An advantage, albeit minute, is a slightly higher capacity served for the proposed reconfiguration in comparison with the complete reconfiguration.

\section{Conclusion}

We stated a new and important problem of itinerant routing in elastic optical networks and proposed a novel connection reconfiguration algorithm. The algorithm achieves the key objective of minimizing the number of new links to configure, which is beneficial for real-time reconfiguration.

We obtained credible simulation results, which suggest the proposed algorithm performs better than a shortest-path reconfiguration algorithm. In comparison with the shortestpath reconfiguration, the proposed algorithm requires half as many new links to reconfigure, and performs comparably in terms of several measured metrics related to establishing and reconfiguring connections, and to the overall network performance.

For these reasons, we argue, the proposed reconfiguration algorithm is indispensable for the itinerant routing in the emerging elastic optical networks, especially in the context of the integration with the next-generation wireless access networks based on the cognitive or cloud radio architectures.

Two general findings for both establishing and reconfiguring a connection are: (a) the optimal routing algorithm for elastic optical networks markedly outperforms the routing with Yen K-shortest paths, and all edge-disjoint paths, and (b) the fittest spectrum allocation policy performs slightly better than the first and random policies.

The proposed reconfiguration algorithm could also be used in link restoration of connections in elastic optical networks, where a connection rerouting is localized to the end nodes of the failed optical link.

Future work could concentrate on the network control for itinerant routing, for example on the trade-offs between the distributed control (itinerant routing can be coordinated autonomously by any node) and the centralized control (itinerant 


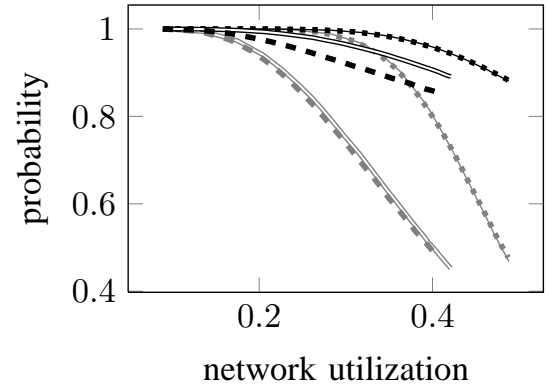

(a) Probability of establishing (gray), and reconfiguring (black) a connection.

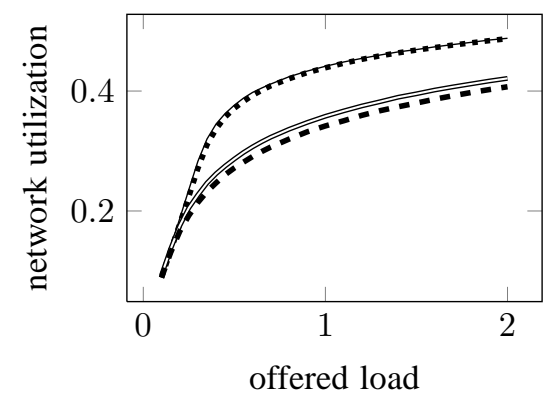

(d) Network utilization.

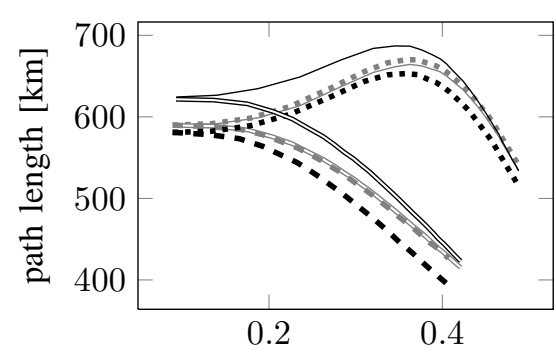

network utilization

(b) Length of an established (gray), and reconfigured (black) connection.

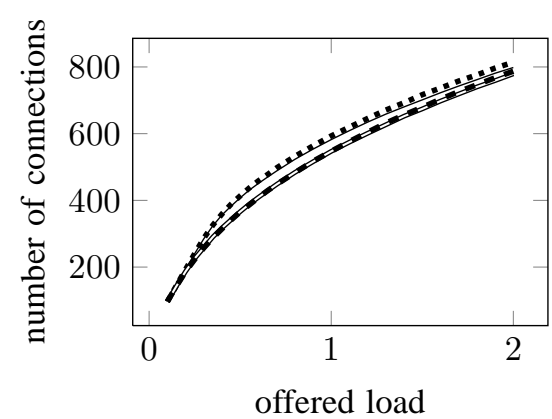

(e) Number of connections

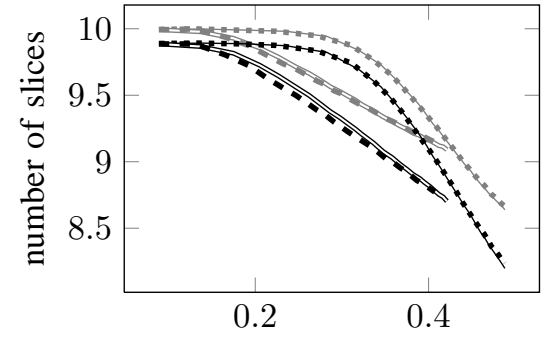

network utilization

(c) Number of slices of an established (gray), and reconfigured (black) connection.

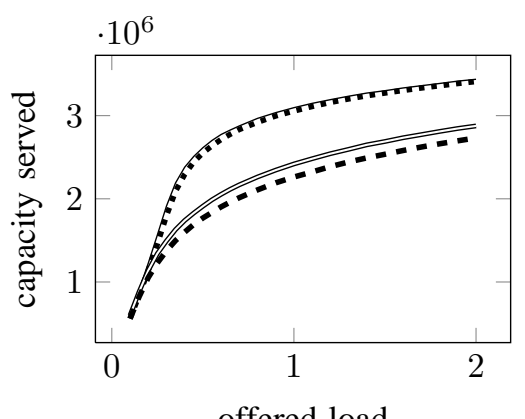

(f) Capacity served.

Fig. 4: Simulation results related to connection and network performance.

routing can be coordinated by a designated node or a set of nodes).

Further research could elaborate on the proposed proactive calculation of candidate reconfiguration paths for the foreseeable events, such as switching a connection to one of the neighboring base stations, or some selected data centers.

\section{REFERENCES}

[1] I. Szcześniak, A. Jajszczyk, and A. Pach, "Mobile routing in elastic optical networks," in IEEE/CIC International Conference on Communications in China, October 2014, pp. 107-111.

[2] B. Skubic, G. Bottari, A. Rostami, F. Cavaliere, and P. Ohlen, "Rethinking optical transport to pave the way for $5 \mathrm{G}$ and the networked society," Journal of Lightwave Technology, vol. 33, no. 5, pp. 1084-1091, March 2015.

[3] N. Bhushan, J. Li, D. Malladi, R. Gilmore, D. Brenner, A. Damnjanovic, R. Sukhavasi, C. Patel, and S. Geirhofer, "Network densification: the dominant theme for wireless evolution into 5G," IEEE Communications Magazine, vol. 52, no. 2, pp. 82-89, February 2014.

[4] V. Eramo, M. Listanti, F. G. Lavacca, R. Sabella, and F. Testa, "Blocking performance of cost efficient integrated OTN/WDM metropolitan networks in static traffic scenario," in Proceedings of the 19th International Conference on Optical Network Design and Modeling (ONDM), May 2015, pp. 204-209.

[5] O. Gerstel, M. Jinno, A. Lord, and S. Yoo, "Elastic optical networking: a new dawn for the optical layer?" IEEE Communications Magazine, vol. 50, no. 2, pp. s12-s20, 2012.

[6] "Spectral grids for WDM applications: DWDM frequency grid," ITU-T Recommendation G.694.1, February 2012.

[7] T. Ohara, M. Teshima, S. Aisawa, and M. Jinno, "OTN technology for multi-flow optical transponder in elastic 400G/1T transmission era," in Optical Fiber Communication Conference and Exposition
(OFC/NFOEC), 2012 and the National Fiber Optic Engineers Conference, March 2012, pp. 1-3.

[8] P. Agyapong, M. Iwamura, D. Staehle, W. Kiess, and A. Benjebbour, "Design considerations for a $5 \mathrm{G}$ network architecture," IEEE Coтmunications Magazine, vol. 52, no. 11, pp. 65-75, Nov 2014.

[9] C.-X. Wang, F. Haider, X. Gao, X.-H. You, Y. Yang, D. Yuan, H. Aggoune, H. Haas, S. Fletcher, and E. Hepsaydir, "Cellular architecture and key technologies for $5 \mathrm{~g}$ wireless communication networks," IEEE Communications Magazine, vol. 52, no. 2, pp. 122-130, February 2014.

[10] I. Szcześniak and B. Woźna-Szcześniak, "Adapted and constrained Dijkstra for elastic optical networks," in 2016 International Conference on Optical Network Design and Modeling (ONDM), May 2016, pp. 1-6.

[11] L. Velasco, A. Castro, M. Ruiz, and G. Junyent, "Solving routing and spectrum allocation related optimization problems: from off-line to inoperation flexgrid network planning," Journal of Lightwave Technology, vol. 32, no. 16, pp. 2780-2795, Aug 2014.

[12] S. Talebi, F. Alam, I. Katib, M. Khamis, R. Salama, and G. N. Rouskas, "Spectrum management techniques for elastic optical networks: a survey," Optical Switching and Networking, vol. 13, pp. 34 - 48, 2014.

[13] A. Szymański, A. Lasoń, J. Rząsa, and A. Jajszczyk, "Grade-of-servicebased routing in optical networks," IEEE Communications Magazine, vol. 45, no. 2, pp. 82-87, Feb 2007.

[14] M. Ali, G. Ellinas, H. Erkan, A. Hadjiantonis, and R. Dorsinville, "On the vision of complete fixed-mobile convergence," Journal of Lightwave Technology, vol. 28, no. 16, pp. 2343-2357, 2010.

[15] N. Pleros, K. Vyrsokinos, K. Tsagkaris, and N. Tselikas, "A $60 \mathrm{GHz}$ radio-over-fiber network architecture for seamless communication with high mobility," Journal of Lightwave Technology, vol. 27, no. 12, pp. 1957-1967, June 2009.

[16] J. Beas, G. Castañon, I. Aldaya, A. Aragon-Zavala, and G. Campuzano, "Millimeter-wave frequency radio over fiber systems: a survey," IEEE Communications Surveys and Tutorials, vol. 15, no. 4, pp. 1593-1619, Fourth Quarter 2013.

[17] M. Zhu, L. Zhang, J. Wang, L. Cheng, C. Liu, and G.-K. Chang, "Radio-over-fiber access architecture for integrated broadband wireless 
services," Journal of Lightwave Technology, vol. 31, no. 23, pp. 36143620, Dec 2013.

[18] E. Cetinkaya, M. Alenazi, Y. Cheng, A. Peck, and J. Sterbenz, "On the fitness of geographic graph generators for modelling physical level topologies," in 2013 5th International Congress on Ultra Modern Telecommunications and Control Systems and Workshops (ICUMT), Sept 2013, pp. 38-45.

[19] I. Szcześniak. (2016, September) The website of the SDI software. [Online]. Available: http://www.irkos.org/sdi 